\title{
Eine Anmerkung zur Kompetenzorientierung
}

\author{
Roland Schröder
}

Sebastian Stiller befragte zwölf Teilnehmer einer Fachtagung zu Ursachen der Mathematik-Lücke zwischen Schule und Hochschule [1]. Vorab eine kurze Auswertung der Antworten:

- Fünf Nennungen

$\triangleright$ Verringerung der Stundenzahl beim Mathematikunterricht (Bach, Koepf, Körner, Schiemann, Walcher)

- Vier Nennungen

\ Inhaltliche Veränderung des Mathematikunterrichts; veränderte Schwerpunktsetzung (Greefrath, Koepf, Walcher, Weidl)

$\triangleright$ Unterschiede im Mathematikverständnis von Schule und Hochschule (Koepf, Körner, Kühnel, Loose)

$\triangleright$ Wissen aus der Mittelstufe zu früh verschüttet; Handwerkszeug wird nicht beherrscht (Koepf, Körner, Krieg, Loose)

- Drei Nennungen

$\triangleright$ Steigender Anteil an Studierenden (Bach, Heinrich, Körner)

$\triangleright$ Heterogenität der Schülerschaft und folglich der Studienanfänger (Heinrich, Koepf, Schiemann)

- Zwei Nennungen

$\triangleright$ Abschaffung von Leistungskursen (Koepf, Schiemann)

- Eine Nennung

$\triangleright$ Technologisch bedingter Grund: CAS, GTR (Körner)

$\triangleright 20$ Jahre Schulreformen (Kühnel)

$\triangleright$ Reizüberflutung (Heinrich)

$\triangleright$ Fehlende beiderseitige Verständigung zwischen Bildungspolitik und Hochschule (Eichler)
Die Ursache „Kompetenzorientierung“, die der Brandbrief ([2]) diagnostiziert, wird nicht ein einziges Mal explizit genannt. Das kann vor allem daran liegen, dass hier ein Sammelbegriff zur Ursache erklärt wurde, was ja sofort berechtigten Widerspruch auslöst. Die Verfasserinnen und Verfasser des Brandbriefs haben aber im Gefolge der Kompetenzorientierung Phänomene erkannt, die durchaus in obigen Antworten enthalten sind:

1. Inhaltliche Veränderung und veränderte Schwerpunktsetzung des Mathematikunterrichts sowie

2. Unterschiede im Mathematikverständnis von Schule und Hochschule.

Schon bei der Suche nach der Antwort auf die Frage Was ist Mathematik? scheiden sich die Geister. Während die einen das Wesen der Mathematik dem Zeitgeist opfern wollen, zieht es andere zurück zu den Wurzeln - von den Babyloniern bis hin zu Leibniz.

Auch bei der Frage Was ist das globale Lernziel von Mathematikunterricht? ist keine Einigung in Sicht, nicht einmal unter den Didaktikerinnen und Didaktikern. Während Lisa Hefendehl-Hebeker ([3]) postuliert, Mathematikunterricht solle erlebbar machen, wie mathematische Wissensbildung geschieht, wollen andere den Sinn der Mathematik bei ihrer Anwendung zur Daseinsbewältigung erkennen. Bei so viel Uneinigkeit kommt der Übergang Schule-Hochschule dann unter die Räder.

Der in der Frage implizit enthaltene Sachverhalt der „Lücke zwischen Schule und Hochschule“ wurde von keinem der Befragten bezweifelt. Darin und auch in den Ant-

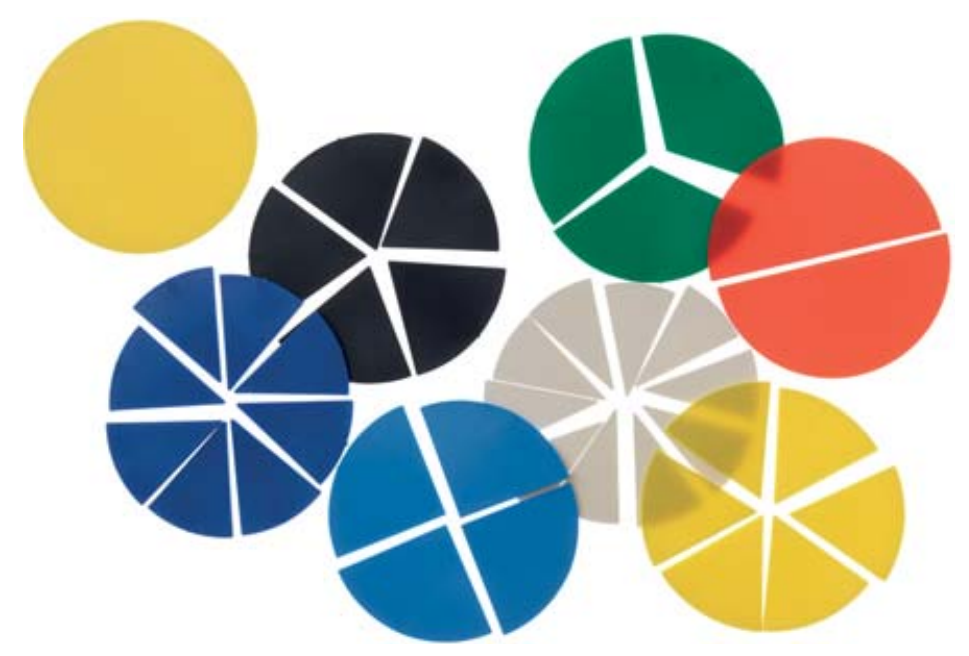


worten auf die hier angesprochene Frage wird im Wesentlichen die Auffassung der Kommission Übergang SchuleHochschule der Verbände DMV, GDM und MNU ([4]) wiedergegeben. Soweit herrscht Einigkeit unter den Fachleuten. Hier ist schon viel, wahrscheinlich alles, gesagt worden. Daher soll nur noch auf diejenigen Ursachen hingewiesen werden, die offenbar in der Fachwelt keinen breiten Konsens finden und die auch nur von wenigen Befragten diagnostiziert wurden.

Anders als in den 196oer bis 199oer Jahren ist heute das Wissen aus der Mittelstufe zu früh verschüttet ([5]) und so steht elementares Handwerkszeug (Bruchrechnung, Gleichungslehre, Termumformung, etc.) schon in der Oberstufe und erst recht im Studium nicht mehr zur Verfügung (vier Nennungen in obiger Auswertung). Dieses Phänomen wurde schon vor der Kompetenzorientierung von vielen Hochschullehrern beschrieben ([5]). In der damit ausgelösten Diskussion prallten Befürworter und Gegner des Einsatzes elektronischer Werkzeuge aufeinander.

Die Diskussion wurde ergebnislos eingestellt und von der neueren Diskussion um die Kompetenzorientierung abgelöst oder überlagert. Ein praktisch erprobtes, wissenschaftlich untermauertes Konzept zur Nutzung elektro- nischer Werkzeuge im Mathematikunterricht wurde nie erstellt. Stattdessen folgt jeder Mathematiklehrer seiner persönlichen Neigung und die Schüler sind dankbar für das Ausbleiben jeglicher Reglementierung. Die Bedeutung des Rechnenlernens beim Erlernen von Mathematik wurde schließlich sogar neu bewertet ([6]). Zum Erlernen von Algebra muss man nun nicht mehr rechnen können?

Der damit charakterisierte technologische Grund erfährt nur eine einzige Erwähnung. Ebenso wie die fehlende beiderseitige Verständigung zwischen Bildungspolitik und Hochschule. Dazu ist festzustellen, dass Verständigung nur stattfinden kann, wenn alle Beteiligten bereit sind, der Argumentation der anderen Seite zu folgen. Die Erfahrung lehrt allerdings, dass die Politik lieber auf die Wirtschaft als auf die Wissenschaft hört.

[1] Mitteilungen der Deutschen Mathematiker-Vereinigung 25-2 (2017), 91-105

[2] tinyurl.com/yd7pvvpr (abgerufen am 29.9.2017)

[3] tinyurl.com/y9grzqgu (abgerufen am 29.9. 2017)

[4] Mitteilungen der DMV 25-2 (2017), 82-88

[5] tinyurl.com/kks5mfy (abgerufen am 29.9.2017)

[6] tinyurl.com/y8qsmbfp (abgerufen am 29.9.2017)

Roland Schröder, Celle

florola@gmx.de

Roland Schröder ist pensionierter Gymnasiallehrer für Mathematik und Sport. Erstes Staatsexamen im Höheren Lehramt in Hannover 1971, zweites Staatsexamen 1973 am Staatlichen Studienseminar Celle, aktiv von 1971 bis 2009, davon 12 Jahre im Auslandsschuldienst (Addis Abeba, Istanbul). Einsatz in der Begabtenförderung Mathematik von 2005 bis 2014.

S. 182 unten: Fraction Circles von Invicta Education, Leicester, England (Foto: Christoph Eyrich) Unten: Calliope Mini, ein Mini-Computer für die Verwendung ab der 3. Klasse

(Foto: Jørn Alraun/CC-BY-SA 4.0)

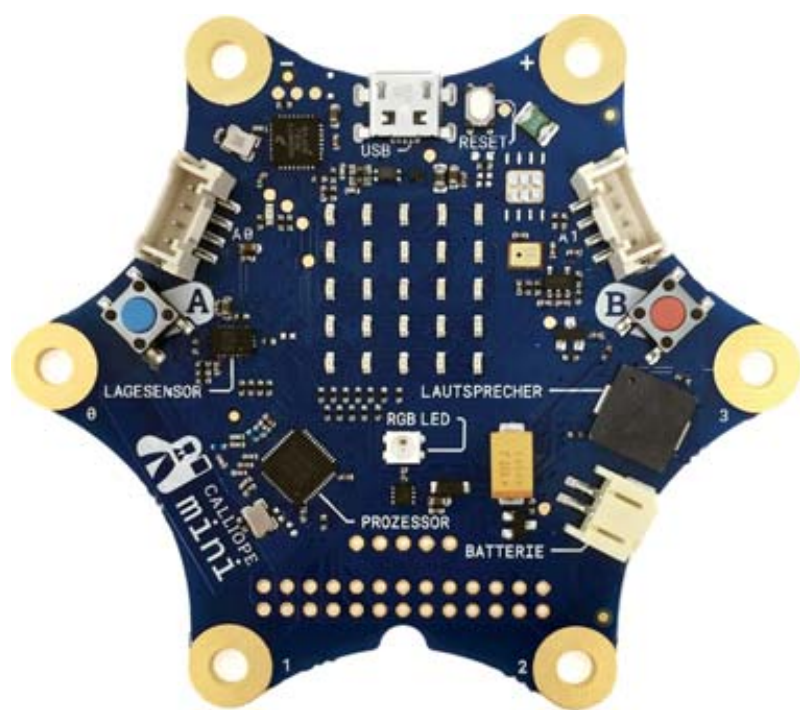

